\title{
An infrared-curtain-based sensor for non-contact recognition of raw-milk gas-liquid two-phase flows
}

\author{
Li Boyi $^{1}$, Cheng Xiaodong ${ }^{2}$ \\ ${ }^{1}$ College of Electronic Information Engineering, Inner Mongolia University, \\ Hohhot, China, 010021 \\ ${ }^{2}$ College of Electronic Information Engineering, Inner Mongolia University, \\ Hohhot, China, 010021 \\ 12Liboyi.imu.edu@outlook.com, ${ }^{2}$ cxd0808@imu.edu.cn
}

Keywords: Signal acquisition and analysis; Infrared sensor; Gas-liquid two-phase flow analysis

\begin{abstract}
A model illustrating the gas-liquid two-phase flow (GLTF) of slug raw-milk in micro tubes was built, and an infrared-curtain-based sensor was designed for non-contact recognition of raw milk in GLTF. An infrared curtain signal acquisition site was set at both upstream and downstream for analysis of time domain, frequency spectrum and cross-correlation. Photos of two-phase flows were taken by a high-speed camera and used as a synchronous control of signal analysis. The analysis of infrared curtain signals shows that the spectral energy of raw-milk GLTF at low-speed condition is concentrated at the low-frequency section, but at high-speed condition, it shifts to the high-frequency section, with two peaks nearby. Therefore, the infrared-curtain-based non-contact sensor succeeded in recognition of raw milk GLTFs in micro tubes. Compared with a contact- typed pressure sensor, this non-contact sensor can utmost reduce the interference on flow patterns. This sensor severs as a theoretical basis for further research on pattern recognition and flowing mechanism of GLTFs. This study also provides some clues for exploration of infrared-curtain-based non-contact computation of raw-milk flow quantity.
\end{abstract}

\section{Introduction}

Gas-liquid two-phase flows (GLTFs) are ubiquitous in the nature and various industries. Because of interface effect, GLTFs will produce different flow patterns, such as slug flow, bubbly flow, and annular flow. So far, detection of GLTFs becomes a concern and hotspot in this researching field [1]. The available detection methods include conductivity sensor [2], pressure sensor, fast valve method, ray method, optical method, and tomographic imaging. However, most of these methods are still at the stage of laboratory research. At present, China's dairy industry is rapidly developing and transiting to the large-scale breeding phase $[3,4,5]$. The problem urgently to be solved at this phase is how to access continuous data about milk production and milking status of cows. These data underlie the analysis of cow health condition. The raw milk extruded from a cow contains bubbles and thus is a GLTF. The existing milk quantity measurement methods include artificial reading from milk recorders, weighing with pressure sensor, and Matlab-based liquid level recognition method. Though these methods are capable of measuring raw milk flow in a two-phase flow, they all require the collection of raw milk into recorders before computation. Therefore, these methods are inadequate for continuous monitoring of milking speed and volume, which does not satisfy the requirement of large-scale production in the future. Raw milk as a food raw material should also meet relevant food hygiene standards. However, the turbines commonly used in liquid detection will directly contact the raw milk, which does not accord with dairy production standards and therefore cannot be used. Liquid recognition in GLTFs has been intensively studied [6], but contact-typed pressure sensors used there cannot be applied into raw milk detection in GLTFs. In terms of continuous efficient computation of raw milk flow rate in GLTFs, the first step is to investigate "non-contact recognition of raw milk in GLTFs". So far, there are few studies about raw milk detection in GLTFs based on infrared methods. Nevertheless, research on raw milk detection 
based on existing infrared detection theories $[7,8,9]$ is feasible by treating raw milk as a two-phase flow. As reported, when infrared curtains were set at outer walls of raw milk transmission micro tubes, the physical phenomenon of raw milk GLTF inside micro tubes was converted to photoelectric signals, which helped to realize the non-contact recognition of raw milk in GLTFs [10,11,12]. Different from some studies about contact-typed detection of GLTFs based on pressure sensors or conductivity sensors $[13,14,15]$, we used a non-contact infrared sensor. Since an infrared curtain sensor does not contact the raw milk, it is ensured that the mode of two-phase flow inside micro tubes will not be changed by the testing equipment. This method can utmost reduce the disturbance on the two-phase flow pattern, and facilitate the recognition of raw milk in two-phase flows.

\section{Theoretical model}

Based on the Eulerian reference system and the theory of unitary continuous medium flow, we built a GLTF model for recognition of raw-milk inside micro tubes. Then the flowing mechanism and characteristics of slug raw milk in GLTFs were studied, and a non-contact method was used to recognize raw milk in GLTFs.

Raw-milk GLTF model based on Eulerian reference system and continuous medium theory.To recognize raw milk in GLTFs, the first step is flow pattern determination and modeling. The elements of GLTF pattern are combinations of different forms of gas and liquid media. GLTFs have been classified from different perspectives $[16,17,18]$. Nevertheless, owing to the diversity in flow conditions, the flow pattern division based on flow structure morphology is faced with some problems. First, most definitions of flow patterns are based on observations, which inevitably introduces interference from subjective factors. Second, no set of unified and accepted definitions has been established regarding the diversity of definitions. Third, the specificity of flow pattern definition can be only qualified, but no quantitative method has been accepted.

The study target is GLTF mixed by raw milk and air, which are produced at the back-end of a milking apparatus. Based on Eulerian reference system and a continuous medium model, the flow patterns of the study target were divided from the perspective of continuity and by referring to the flow media based on multi-phase flows. In a continuous medium model, an absolutely gap-free continuous medium is composed of an infinite number of infinitesimal, closely-attached and continuous fluid mass points. The increment of fluid mass in the control body is obviously equal to the difference of fluid masses entering and leaving the control body at the same period. In other words, the increment follows a unitary continuous medium flow equation. Figure 1 shows the horizontal flow of GLTFs in micro tubes produced at back-end of the milking machine, and is photographed by the high-speed camera. In this figure, the dark part is the liquid segment, the white part is gas. The gas-liquid segments are very obviously separated, while the lengths of the liquid segments change randomly.

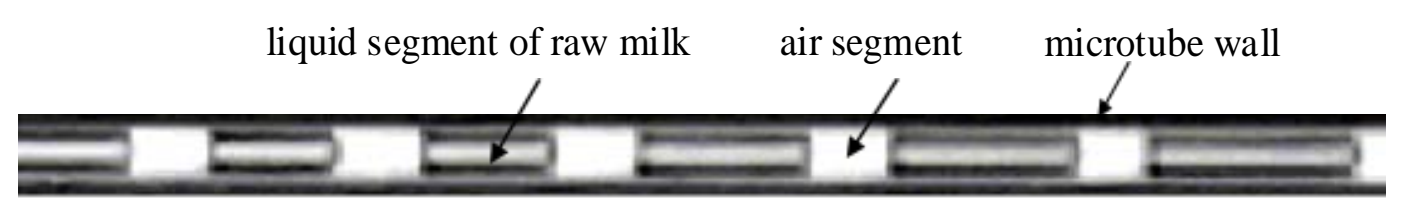

Figure 1 Slug GLTF in microtube produced at back-end of milking machine and photographed by high-speed camera

Thus, the slug GLTF formed by raw milk and gas was used as the study target. The raw-milk GLTF model inside micro tubes is showed in Fig. 2. Specifically, the liquid segments containing several ingredients were idealized to a mono-density liquid. Based on the continuous medium model, we ignored the intermolecular gaps inside the liquid segments, the deformation details at the liquid-gas interface, and the resistance on liquid imposed by tube walls. Figure 2 shows the raw-milk GLTF model inside micro tubes. The liquid segments and gas segments in continuous raw milk appear randomly. The dark part is the liquid segment, the white part is gas. The flowing 
direction is horizontal.

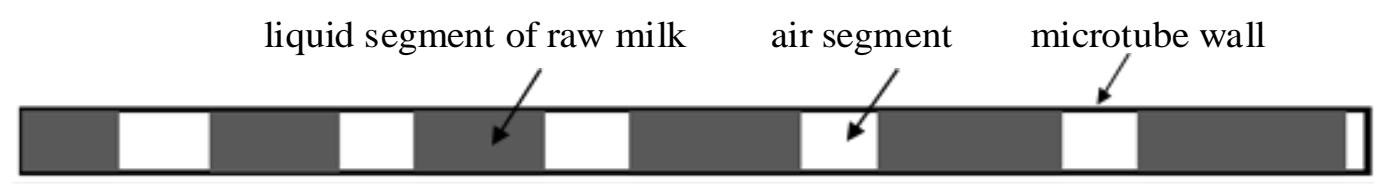

Figure 2 A slug raw-milk GLTF model in micro tubes

In this model, because of compressibility of gas, the lengths of gas segments after long-distance flow in a micro tube will change, and the on-way state parameters will also change, leading to changes of GLTF pattern during flowing process [19]. Such changes will induce changes in forms of liquid segments passing through upstream and downstream, which will severely interfere with the raw-milk recognition. Thus, two infrared curtains with minute length $L$ were respectively installed at the upstream and downstream of the micro tube's outer walls. According to the theory of random signal, when a two-phase flow passes through the infrared curtains within $L$, its flow pattern is basically unchanged, which guarantees that the same liquid segment is recognized at the two infrared curtains, thus reducing recognition error.

Based on Beer-Lambert Law, the relationships of the ratio of $I / I_{o}$ ( $I$ : light intensity after passing through a micro tube; $I_{o}$ : incident light intensity) with concentration of absorbing material $c$, thickness $d$, and extinction coefficient $k$ were used. When $I_{o}$ is constant, the light intensity in the infrared receiving sensor after passing through raw milk or gas will produce different voltage signals. On this basis, non-contact raw-milk recognition in GLTFs can be realized.

Signal analysis based on theory of random signal cross-correlation.Infrared curtains were set at the micro tube's outer walls and their signal receiving terminals produced two-phase flowing random signals $x(t)$ and $y(t)$ respectively, Usually, the cross-correlation degree is expressed as a cross-correlation coefficient:

$$
\rho_{x y}(\tau)=\frac{\lim _{T \rightarrow \infty} \frac{1}{T} \int_{0}^{T} x(t) y(t+\tau) d t-\mu_{x} \mu_{y}}{\sigma_{x} \sigma_{y}}
$$

Normally, correlation degree can be determined from the range of the cross-correlation coefficient: 0.6-1.0 (high), 0.4-0.6 (medium), 0.2-0.4 (weak), and 0.0-0.2 (very weak or no correlation).

\section{Experimental}

In the experiments, raw-milk GLTFs were introduced into micro tubes. Two infrared curtains were set at upstream and downstream respectively. Data were acquired with Labview high-performance data acquisition cards, and then sent into analysis of time domain and frequency spectrum on NI Signal Express and Matlab. The cross-correlation between signals was analyzed using the theory of random signal cross-correlation. The GLTFs were also photographed with a high-speed camera for analysis.

Closed-loop horizontal GLTF experimental platform.Figure 3a shows the sketch map of the raw milk GLTF experimental platform. To achieve the GLTF signal, we designed a closed-loop horizontal GLTF experimental loop. In particular, 1-raw milk tank, 2-air tank, 3-air compressor, 4 and 5-control valves, 6-microtube detection segment (quartz glass micro tubes, infrared curtains, signal acquisition circuit, high-speed camera), 7-gas flowmeter, 8-liquid flowmeter, 9-gas-liquid mixer, and 10-pumps. The experimental platform is shown in Figure $3 \mathrm{~b}$. 

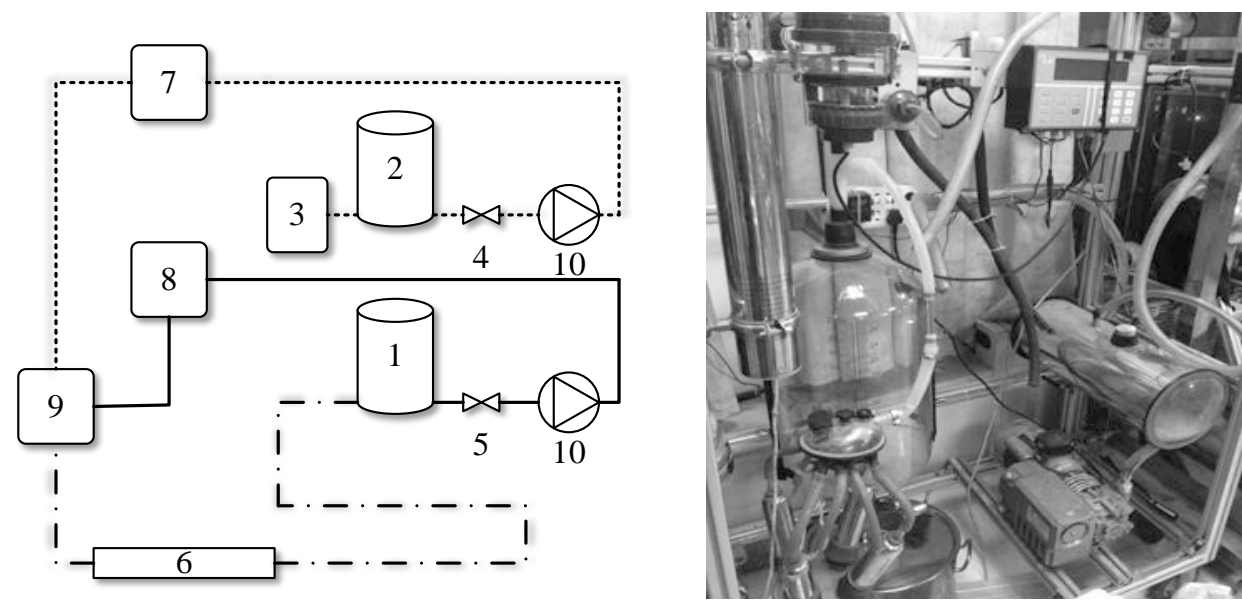

Fig. 3 Experiment platform. (a) Schematic diagram; (b) Experiment platform

The micro tube's detection section is installed with infrared curtains (schematic diagram in Fig. 4a). The infrared curtains are placed outside quartz micro tubes (outer diameter $10.0 \mathrm{~mm}$; inner diameter $6.0 \mathrm{~mm}$ ). The two-phase flow moves horizontally, where $\mathrm{T} 1$ and $\mathrm{R} 1$ constitute the upstream infrared curtain, while T2 and R2 constitute the downstream infrared curtain, T1 and T2 are the irradiant two-phase flow from the infrared transmitting terminals, R1 and R2 are the voltage signals converted from the infrared signals when $\mathrm{T} 1$ and $\mathrm{T} 2$ from the infrared receiving terminal are blocked by the two-phase flow. The physical diagram is showed in Fig. 4b. A flow moving inside a tube will suffer from various random disturbances. Based the theory of random signal cross-correlation, within short distance $L$, we believe that the flow noise signals are consistent. Thus, the measurement precision based on this theory is basically not affected by fluid temperature, concentration or medium components. In the experiments, $L$ was $=20.0 \mathrm{~mm}$.
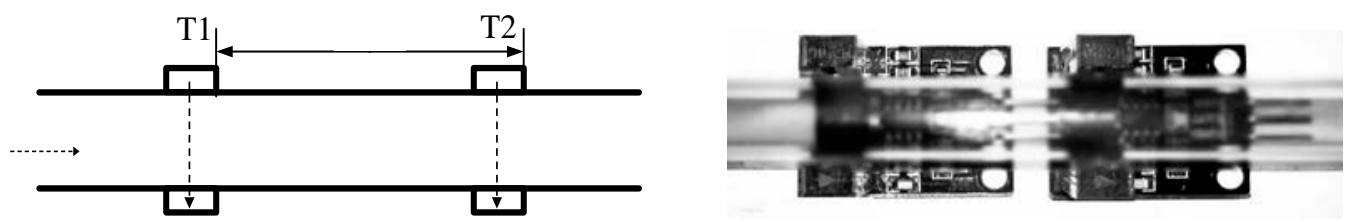

Fig. 4 Infrared curtains. (a) Schematic diagram; (b) Physical diagram

Signal data acquisition and analysis platform. In this study, signals from the receiving terminals of several infrared curtains are involved in signal acquisition, recording and analysis. Two problems should be solved: synchronous input and real-time synchronous recording of multi-route signals. For this reason, a data acquisition and analysis platform was set at the micro tube's detection segment. The overall architecture is shown in Figure 5. The upstream and downstream infrared curtains were each connected with a signal pretreatment circuit and then each connected into a DAQ USB-6251 collection card (US National Instruments). Then a data acquisition record program was compiled on Labview for realizing the communication between PC and the acquisition card, and for synchronous acquisition and real-time recording of multichannel infrared curtain signals. Data analysis was conducted on Matlab. We also used a high-speed camera (Sony NEX-FS700CK) for the high-speed synchronously photography. This platform is able to synchronously trace and record multichannel data, and to synchronously input 16-channel simulated or digital signals. This platform avoids repeated data output during offline analysis, ensures precise data acquisition and significantly accelerates data analysis efficiency. 


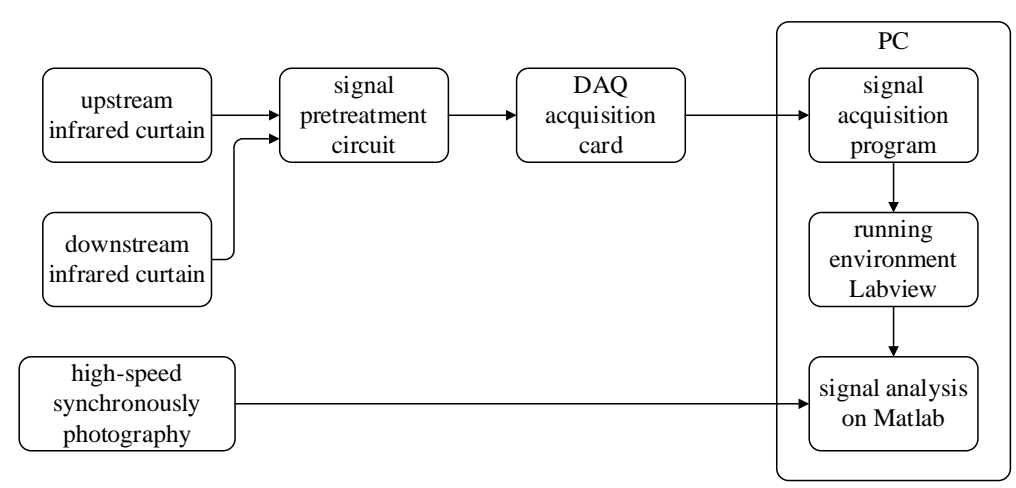

Fig. 5 Structure of the signal data acquisition and analysis platform

\section{Signal processing and experimental results}

The low-speed and high-speed states of GLTF were comparatively tested. The logic level of infrared curtains was set with a high level above $2 \mathrm{~V}$ when raw milk passes through the infrared curtain and at $0 \mathrm{~V}$ when no raw milk passes. The infrared transmitting terminals $\mathrm{T} 1$ and $\mathrm{T} 2$ are supplied with DC constant voltage and emit constant-intensity signals. The signals acquired were introduced into Matlab and the results are listed below.

The low-speed raw-milk GLTF produces time-domain signals at the upstream and downstream infrared curtains as showed in Figs. 6a and 6b, respectively. The images in Figs. 6a and 6b were processed with fast Fourier transform (FFT), and the spectra are showed in Figs. 6c and 6d, respectively. When a two-phase flow moves from a position in the upstream to another position in the downstream, signal fluctuation may occur. Thus, the cross-correlation coefficient between two signals from the upstream and downstream respectively should be quantified. The results of cross-correlation coefficient are showed in Fig. 6e. At this status, the photography of the two-phase flow acquired by the high-speed camera are showed in Fig. 6f.
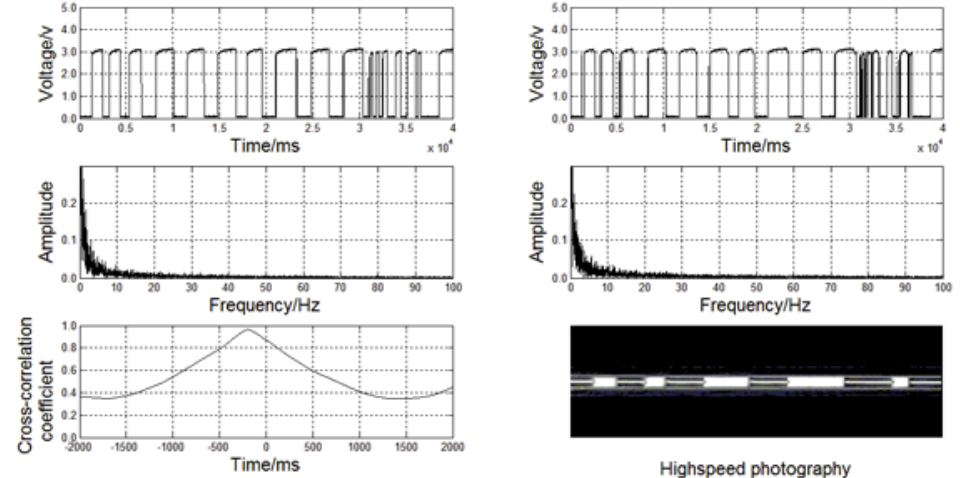

Highspeed photography

Figure 6 The signal analysis of gas-liquid two-phase flow of raw milk in low speed (a)Upstream of the time-domain analysis of infrared light curtain; (b) Downstream of the time-domain analysis of infrared light curtain; (c) Upstream of the spectrum analysis of infrared light curtain; (d) Downstream of the spectrum analysis of infrared light curtain; (e) Signals cross-correlation analysis; (f) High-speed photography image;

The high-speed raw-milk GLTF produces time-domain signals at the upstream and downstream infrared curtains as showed in Figs. 7a and 7b, respectively. The images in Figs. 7a and 7b were treated with FFT, and the spectra are showed in Figs. 7c and 7d, respectively. When a two-phase flow moves from a position in the upstream to another position in the downstream, signal fluctuation may occur. Thus, the cross-correlation coefficient between two signals from the upstream and downstream respectively should be quantified. The results of cross-correlation coefficient are showed in Fig. 7e. At this status, the photography of the two-phase flow acquired by the high-speed camera are showed in Fig. $7 f$. 

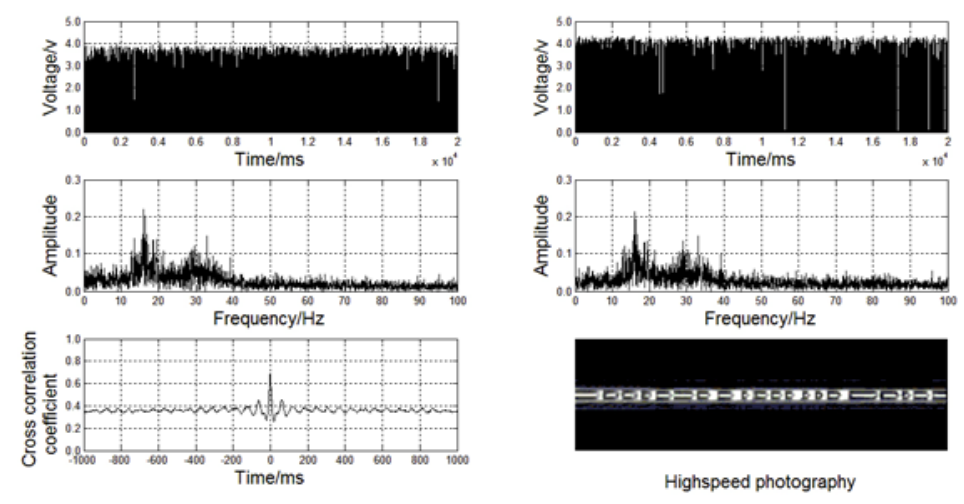

Figure 7 The signal analysis of gas-liquid two-phase flow of raw milk in high speed (a) Upstream of the time-domain analysis of infrared light curtain; (b) Downstream of the time-domain analysis of infrared light curtain; (c) Upstream of the spectrum analysis of infrared light curtain; (d) Downstream of the spectrum analysis of infrared light curtain; (e) Signals cross-correlation analysis; (f) High-speed photography image;

\section{Discussion and analysis}

1. Based on the time-domain signal images in Figs. 6a and 6b, at very low flow speed, the high and low levels change very slowly, indicating that the liquid segment and gas segment in the raw milk GLTF are very long and the two-phase variation is very slow, which are consistent with Fig. $6 \mathrm{f}$ acquired by the high-speed camera.

2. Based on the time-domain signal images in Figs. 7a and 7b, at very high flow speed, the high and low levels change very quickly, indicating that the liquid segment and gas segment in the raw milk GLTF assume are very short and the two-phase variation is very rapid, which are consistent with Fig. $7 f$ acquired by the high-speed camera.

3. Based on Figs. 6e and 7e, the cross-correlation coefficients between upstream and downstream signals $x(n)$ and $y(n)$ produced at low-speed and high-speed are 0.97 and 0.69 , respectively, which both fall within 0.6-1.0, indicating high correlation degrees. These results are consistent with Figs. 6a-b and Figs. 7a-b, respectively. Therefore, the preset distance of $20 \mathrm{~mm}$ between infrared curtains satisfies the condition that the flowing signals in a micro-displacement are consistent, according to the theory of random signal cross-correlation. These results indicate that the upstream and downstream infrared curtains recognized the liquid segment from the same-status raw-milk two-phase flow at different positions.

4. As showed from the signal frequency-domain images in Figs. 6c-d, the spectral energy at very low flow speed is concentrated at the low frequency $0-10 \mathrm{~Hz}$. The main reason is that the gas segment and the liquid segment of a low-speed two-phase slug flow moving inside a microtube appear slowly and alternatively. These results are consistent with the time-domain images in Figs. $6 a-b$ and the photo acquired by the high-speed camera in Fig. $6 f$.

5 As showed from the frequency-domain images in Figs. 7c-d, the spectral energy at very high flow speed is changed and generally transits from low-frequency to high-frequency. As a result, two single-value peaks appear at the low-frequency section (around $16 \mathrm{~Hz}$ ) and the high-frequency section (around $29 \mathrm{~Hz}$ ), respectively. The appearance of the two single-value peaks can be explained as follows: when a high-speed two-phase slug flow moves in a micro tube, the gas segments and the liquid segments appear alternatively, and the signal energy is concentrated at the photoelectric signal fluctuation, which is produced when a liquid segment passes through an infrared curtain. Moreover, the flow process is also accompanied with abundant fine bubbles, and thus, a part of energy is distributed at the high-frequency section around $29 \mathrm{~Hz}$. These facts explain the appearance of two peaks. 


\section{Conclusions}

With use of infrared curtains, the flow status of raw-milk GLTF inside micro tubes was converted to voltage signals that were feasible for analysis and processing. An experimental platform was built for processing and analysis of raw-milk GLTF signals. Then raw-milk GLTF at low-speed or high-speed were tested with time-domain analysis, frequency analysis, cross-correlation analysis, and observation with high-speed photography. Results show that when signals of raw-milk GLTF changed from low-speed to high-speed, the frequency spectra shifted from low-frequency to high-frequency and showed two peaks. The cross-correlation coefficient between signals from two curtains is from 0.69 to 0.97 , indicating high correlation. Therefore, an infrared curtain-based method for non-contact recognition of raw milk in GLTFs is built successfully. This is a new effective method for research on raw-milk GLTFs. However, further investigation is also required to do much more experiments, such as control experiments at several quantitative flow speeds should be performed for data fitting and analysis.

\section{Acknowledgement}

This work was supported by the Inner Mongolia graduates researching innovation projects (Nos. 1402020201261).

\section{References}

[1] Zhao J Y, Jin N D 2012 Acta Phys. Sin. 614701

[2] Dong F, Jin N D, Zong Y B, Wang Z Y 2008 Acta Phys. Sin. 576146

[3] Liu F, Wei W, He Z W 2014 World Agriculture 173

[4] Li X C 2013 Contemporary Animal Husbandry 62

[5] Liu Q M, Zhang J G, Huo X J, Wang H, Li J G 2011 Journal of Agricultural Mechanization 279

[6] Sun B,Wang E P,Zheng Y J 2011 Acta Phys. Sin. 6014701

[7] He F, Yan Z H, Xie G J,Zhang J G, Gong J H, Xu B, Wang Y S 2013 Manned Spaceflight 1958

[8] Yan Z H, He F, Xie G J, Zhang J G, Xiao Y W, Yuan Y H, Song Z Y 2012 Micro Processors 381

[9] Gong J H, Liang F C, Liu D X, Yan H 2012 Journal of Zhengzhou University of Light Industry(Natural Science) 279

[10] Zuo Z L, Zheng B, Gan L, Deng Y, Bai F M 2011 Journal of Engineering Design 18149

[11] Xiong J X, Chen P 2000 Journal of Changchun Institute of Optics and Fine Mechanics 2312

[12] Kawaji M 2007 Proceedings of American Society of Mechanical Engineering 5 th International Conference on Nanochannels Microchannels and Minichannels Puebla, Mexico June, 2007 p1-6

[13] Duan R X, Yu D, Wu H H, Gong J, Hu S W, Zhou T, Zheng L J 2012 Experimental Technology and Management 2962

[14] Liang F C, Wang D, Lin Z H 2008 Journal of Xi'an Jiaotong University 4255

[15] Liang F C, Chen Q, Wang D, Lin Z H 2008 Journal of China University of Petroleum 3295

[16] Spedding P L, Spenee D R 1993 Multiphase Flow 19280

[17] Oshinowo T, Charles M B 1974 Chemical Engineering Science 5235

[18] Hoogendoorn C J 1959 Chemical Engineering Science 917

[19] Zhao D X, Cao X W, Zhang Y H, Cui M W, Zhang N 2014 Oil \& Gas Storage and Transportation 2167 